\title{
Assessment of Radioactivity Levels of Foodstuffs Entering Mombasa Port, Kenya
}

\author{
Michael Atogo \\ Department of NPP Engineering, KEPCO International Nuclear Graduate School, Haemaji-ro, Ulju-gun, 45014Ulsan, Korea
}

Received: July 22, 2016 / Accepted: August 09, 2016 / Published: November 30, 2016.

\begin{abstract}
Evaluation of radionuclide concentrations in foodstuffs is of great concern owing to the potential radiological hazards to human health. This study was carried out to assess the radionuclide concentrations of ${ }^{40} \mathrm{~K},{ }^{134} \mathrm{Cs},{ }^{137} \mathrm{Cs},{ }^{131} \mathrm{I},{ }^{90} \mathrm{Sr},{ }^{239} \mathrm{Pu}$, in selected foodstuffs, in order to assess the effects of the elevated levels of radionuclides concentration in environmental media after the FDNPS (Fukushima Daiichi Nuclear Power Station) accident on imported food. HPGe (high purity germanium) detector based spectrometry system coupled with multi-channel analyzer was used for the assessment. The mean concentrations of ${ }^{40} \mathrm{~K},{ }^{134} \mathrm{Cs},{ }^{137} \mathrm{Cs}$, ${ }^{131} \mathrm{I},{ }^{90} \mathrm{Sr},{ }^{239} \mathrm{Pu}$, were found to be $50.1 \pm 2.44 \mathrm{~Bq} / \mathrm{Kg}, 109.6 \pm 0.141 \mathrm{~Bq} / \mathrm{Kg}, 134.2 \pm 0.027 \mathrm{~Bq} / \mathrm{Kg}, 2.57 \pm 0.019 \mathrm{~Bq} / \mathrm{Kg}, 0.59 \pm 0.03$ $\mathrm{Bq} / \mathrm{Kg}, 0.160 \pm 0.033 \mathrm{~Bq} / \mathrm{Kg}$, and $0.227 \pm 0.021 \mathrm{~Bq} / \mathrm{Kg}$ respectively. The analysis showed that the radioactivity levels of ${ }^{241} \mathrm{Am}$, ${ }^{134} \mathrm{Cs}$ and ${ }^{137} \mathrm{Cs}$ were found to be relatively low and within the acceptable levels.
\end{abstract}

Key words: Radioactivity, radionuclides, foodstuffs.

\section{Introduction}

Radionuclide concentration measurements in foodstuffs and environmental samples have recently received a great deal of attention owing to their potential to cause harmful effects to human health. As stated in Ref. [1], radionuclides are naturally present in environment, which includes our bodies, food and water. Man is exposed to radiation (also known as background radiation) from these radionuclides on a daily basis [2]. The radiation comes from cosmic rays as well as from NORM (naturally-occurring radioactive materials) found in the soil, water and air. People can also be exposed to artificial radiation that emanates from man-made activities such as agriculture, research, medical diagnostic intervention and from the use of nuclear energy or irradiation of materials.

However, exposure to ionizing radiation can be harmful, and the degree of harm to human health depends on the type of radionuclides and the length of

Corresponding author: Michael Atogo, M.S., radiation protection officer, research field: environmental radioactivity. time people are exposed to it [3]. The possible exposure pathways to individuals include direct radiation from an airborne radioactive plume, inhalation of radioactive gases and aerosols, external radiation from contaminated ground, and internal dose from contaminated food. Patra et al. [4] demonstrate how previous studies on internal doses from ingestion of foodstuffs that are contaminated with radioactive sources, have shown that food intake is one of the important pathways through which man is exposed to artificial radioactivity which may have considerable health concerns. In addition, radioactive compounds that accumulate in the food chain, such as radioisotopes of iodine, cesium and strontium dominate in delivering doses to humans and biota [5].

In this study, radionuclides of major importance in the contamination of food and environmental samples were analyzed to determine the level of radioactivity in selected imported foodstuffs. As listed in Ref. [6], these include ${ }^{40} \mathrm{~K},{ }^{134} \mathrm{Cs},{ }^{137} \mathrm{Cs},{ }^{131} \mathrm{I},{ }^{90} \mathrm{Sr},{ }^{239} \mathrm{Pu}$, and the objective was to investigate whether there were elevated levels of radionuclides in the foodstuffs 
entering the port following the Fukushima-Daichii NPP accident in Japan.

\section{Materials and Methods}

This study was carried out to assess the levels of radioactivity in selected foodstuffs that enter the port of Mombasa. The methodology used presents experimental setup, preparation, sealing and measurement of food samples that were collected, and then analyzed to determine the radionuclide concentrations in the samples.

Foodstuffs were selected according to their importation volume and the consumption practices of the local population residing in the city. Thirteen samples of seven different kinds of imported foodstuff were collected for analysis.

The sample types and their countries of origin are listed in Table 1. Beef samples were washed, and the non-edible parts were removed. They were weighed, freeze dried and then homogenized. $500 \mathrm{~g}$ from each sample was packed in a marinelli beaker, and sealed for 28 days to allow for radioactivity equilibrium between parents and their daughter radionuclides [7].

Rice, milk powder and sugar were put and sealed in a marinelli beaker without any pre-treatments. The other samples were washed with ultrapure water to remove soil and dust particles, and then put in a furnace for $24 \mathrm{~h}$ at $300{ }^{\circ} \mathrm{C}$ for ashing (preconcentration). Ash samples were powdered, homogenized, packed and sealed for four weeks in a marinelli beaker.

All samples were measured by gamma spectrometry system, manufactured by Canberra, using an HPGe (high purity germanium) (Canberra GX4020) with the resolution $1.9 \mathrm{KeV}$, energy range above $3 \mathrm{KeV}$ and relative efficiency $42 \%$, as described in Ref. [8]. The detector and pre-amplifier were placed inside the shield and cooled by liquid nitrogen from a vertical dipstick cryostat $(7500 \mathrm{SL})$. The integrated signal processor consists of a pulse height analysis system to transform pulse, which are finally collected by a computer based MCA (multi-channel analyzer). The set-up was as shown in Fig. 1.

\section{Results and Discussion}

The seven different types of imported foodstuffs were analysed to determine the activity of radionuclides namely: ${ }^{40} \mathrm{~K},{ }^{90} \mathrm{Sr},{ }^{131} \mathrm{I},{ }^{239} \mathrm{Pu},{ }^{241} \mathrm{Am}$, ${ }^{134} \mathrm{Cs}$ and ${ }^{137} \mathrm{Cs}$. Analysis of the data presented in Table 1 shows that the average activity level of the ${ }^{131} \mathrm{I}$

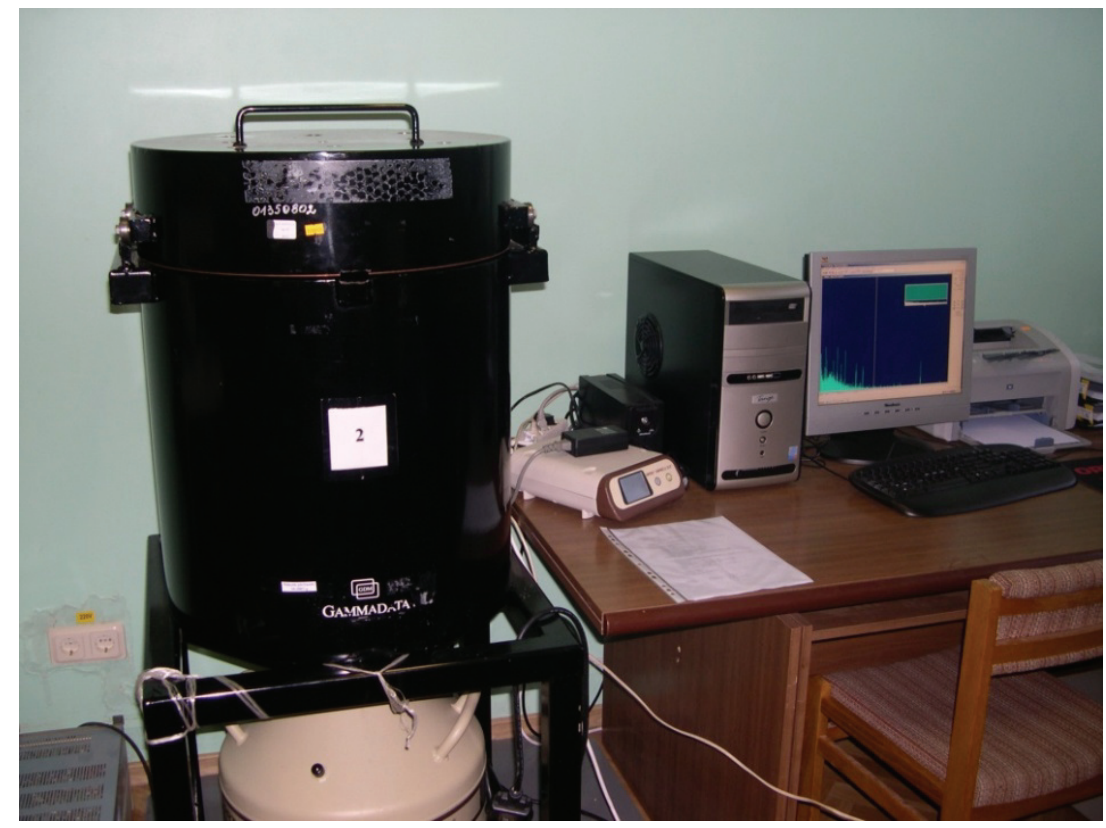

Fig. 1 Gamma spectroscopy equipment. 
was remarkably higher compared to guideline Levels on radionuclides contained in food issued by United Nations FAO/WHO [2]. Column 3 of Table 1 suggests that the activity concentrations of ${ }^{131} \mathrm{I}$ in the various foodstuffs studied vary from $109.7 \pm 0.050$ to $153 \pm$ $0.007 \mathrm{~Bq} / \mathrm{Kg}$ with a mean value of $134.20 \pm 0.027$ $\mathrm{Bq} / \mathrm{Kg}$.

The average activity concentrations of the 13 food samples studied are shown in Fig. 2. All the foodstuffs except sugar showed a relatively high activity concentration of the naturally occurring radioactive radionuclide ${ }^{40} \mathrm{~K}$ with an average activity concentration of $50.1 \pm 2.44 \mathrm{~Bq} / \mathrm{Kg}$. The highest activity of this radionuclide was found in beef and the lowest activity was found in sugar.

The concentrations of ${ }^{40} \mathrm{~K}$ and ${ }^{137} \mathrm{Cs}$ in the mentioned imported foodstuffs were less than, or comparable with levels of the local foodstuffs previously studied, except for ${ }^{40} \mathrm{~K}$ concentration in milk powder and rice which were higher.

Radioactivity levels of ${ }^{241} \mathrm{Am},{ }^{134} \mathrm{Cs}$ and ${ }^{137} \mathrm{Cs}$ were found to be relatively low and within the acceptable levels required by the food and environmental radiation regulations in Kenya.

Table 1 Radioactivity levels of samples of imported foodstuffs, $(\mathrm{Bq} / \mathrm{Kg})$.

\begin{tabular}{llllllllll}
\hline No. & Sample & $\begin{array}{l}\text { Origin } \\
\text { country }\end{array}$ & ${ }^{40} \mathrm{~K}$ & ${ }^{90} \mathrm{Sr}$ & ${ }^{131} \mathrm{I}$ & ${ }^{239} \mathrm{Pu}$ & ${ }^{241} \mathrm{Am}$ & ${ }^{134} \mathrm{Cs}$ & ${ }^{137} \mathrm{Cs}$ \\
\hline 1 & Beef & Australia & $113.0 \pm 3.2$ & $128.6 \pm 0.013$ & $153.2 \pm 0.007$ & $3.00 \pm 0.023$ & $0.78 \pm 0.001$ & $0.074 \pm 0.034$ & $0.089 \pm 0.003$ \\
2 & Beef & Australia & $107.0 \pm 3.1$ & $125.7 \pm 0.011$ & $149.5 \pm 0.006$ & $2.95 \pm 0.023$ & $0.80 \pm 0.001$ & $0.077 \pm 0.032$ & $0.143 \pm 0.002$ \\
3 & Salmon & Japan & $104.5 \pm 3.0$ & $125.6 \pm 0.012$ & $150.7 \pm 0.006$ & $2.65 \pm 0.024$ & $0.76 \pm 0.002$ & $0.065 \pm 0.034$ & $0.241 \pm 0.003$ \\
4 & Salmon & Japan & $104.2 \pm 3.4$ & $123.5 \pm 0.012$ & $151.5 \pm 0.006$ & $2.64 \pm 0.025$ & $0.75 \pm 0.002$ & $0.068 \pm 0.032$ & $0.243 \pm 0.004$ \\
5 & Dried mashroom & China & $101.6 \pm 3.5$ & $113.9 \pm 0.013$ & $132.6 \pm 0.015$ & $3.00 \pm 0.030$ & $0.43 \pm 0.001$ & $0.216 \pm 0.033$ & $0.265 \pm 0.004$ \\
6 & Dried mashroom & China & $101.8 \pm 3.4$ & $112.7 \pm 0.014$ & $133.9 \pm 0.008$ & $2.90 \pm 0.026$ & $0.45 \pm 0.002$ & $0.263 \pm 0.030$ & $0.267 \pm 0.003$ \\
7 & Sugar & Brazil & $1.9 \pm 0.2$ & $110.0 \pm 0.011$ & $132.6 \pm 0.006$ & $2.47 \pm 0.003$ & $0.61 \pm 0.004$ & $0.216 \pm 0.047$ & $0.284 \pm 0.055$ \\
8 & Sugar & Brazil & $1.8 \pm 0.3$ & $112.3 \pm 0.013$ & $131.9 \pm 0.045$ & $2.45 \pm 0.004$ & $0.62 \pm 0.002$ & $0.218 \pm 0.046$ & $0.284 \pm 0.030$ \\
9 & Rice & Pakistan & $32.5 \pm 1.3$ & $109.6 \pm 0.210$ & $134.0 \pm 0.038$ & $2.43 \pm 0.001$ & $0.65 \pm 0.003$ & $0.179 \pm 0.042$ & $0.241 \pm 0.028$ \\
10 & Rice & Viet Nam & $31.8 \pm 1.5$ & $110.7 \pm 0.300$ & $132.5 \pm 0.042$ & $2.45 \pm 0.002$ & $0.64 \pm 0.003$ & $0.185 \pm 0.025$ & $0.240 \pm 0.032$ \\
11 & Dried maize & South Africa & $26.5 \pm 3.1$ & $89.0 \pm 0.455$ & $110.8 \pm 0.055$ & $1.98 \pm 0.025$ & $0.46 \pm 0.006$ & $0.175 \pm 0.028$ & $0.145 \pm 0.030$ \\
12 & Dried maize & South Africa & $26.6 \pm 2.8$ & $86.4 \pm 0.350$ & $109.7 \pm 0.050$ & $2.00 \pm 0.030$ & $0.43 \pm 0.005$ & $0.173 \pm 0.026$ & $0.143 \pm 0.030$ \\
13 & Milk powder & Australia & $24.3 \pm 2.9$ & $76.4 \pm 0.425$ & $121.6 \pm 0.065$ & $2.50 \pm 0.030$ & $0.32 \pm 0.004$ & $0.176 \pm 0.020$ & $0.365 \pm 0.055$ \\
\hline
\end{tabular}

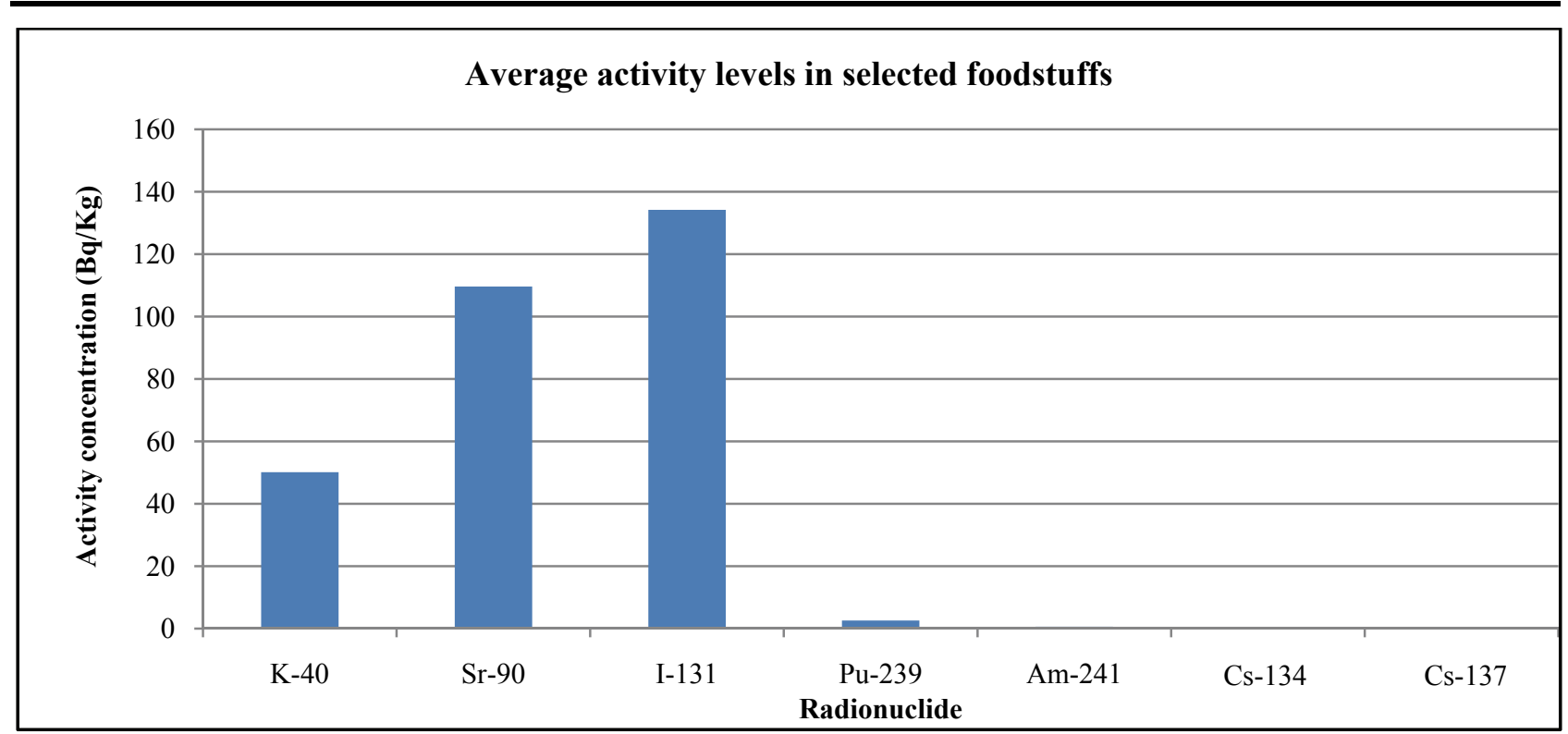

Fig. 2 Average activity concentrations of radionuclides in foodstuffs. 


\section{Conclusions}

Radionuclide concentrations in imported foodstuffs widely consumed in Kenya have been determined. ${ }^{137} \mathrm{Cs}$ was detected in low levels in the foodstuffs considered. The study revealed the possibility of elevated levels of the radionuclide contents in food originating from regions contaminated with radionuclides in the various pathways.

\section{Acknowledgement}

This research was supported by the 2016 Research Fund of the KEPCO International Nuclear Graduate School (KINGS), Republic of Korea.

\section{References}

[1] NNR, South Africa, Understanding Radiation. Accessed May 13, 2016. http://www.nnr.co.za/understanding-radiation-2/.

[2] INFOSAN. 2011. "Information on Nuclear Accidents and Radioactive Contamination of Foods.” International Food Safety Authorities Network, WHO/FAO. http://www.who.int/foodsafety/fs_management/radionucl ides_and_food_300311.pdf.

[3] Spanish Food Safety and Nutrition Agency. Accessed April 23, 2016. http://foodsafetycheese.com/.

[4] Patra, A. C., Mohapatra, S., Sahoo, S. K., Lenka, P., Dubey, J. S., Thakur, V. K., Kumar, A. V., Ravi, P. M., and Tripathi, R. M. 2014. "Assessment of Ingestion Dose Due to Radioactivity in Selected Food Matrices and Water near Vizag, India." Journal of Radioanalytical and Nuclear Chemistry 300 (3): 903-10.

[5] Pavel, P. P., and Katsumi, H. 2015. "Fukushima Radionuclides in the NW Pacific, and Assessment of Doses for Japanese and World Population from Ingestion of Seafood." Scientific Reports 5: 9016. doi: 10.1038/srep09016.

[6] Food Monitoring: Note Nuclear Spectroscopy Systems, file://C:/Users/Administrator/Downloads/Radioactivity-a nd-its-Measurements-in-Foodstuffs\%20(2).pdf.

[7] Hosseini, T., Fativand, A. A., Barati, H., and Karimi, M. 2006. "Assessment of Radionuclides in Imported Foodstuffs in Iran." International Journal of Radiation Research 4 (3): 149-53.

[8] Mollah, A. S. Assessments of Internal Doses by Ingestion of Radioactive Foodstuffs in Bangladesh. 